\section{Transfer of Fas (CD95) protein from the cell surface to the surface of polystyrene beads coated with anti-Fas antibody clone $\mathrm{CH}-11$}

\author{
Hirofumi Sawai, Naochika Domae \\ Department of Internal Medicine, Osaka \\ Dental University, Hirakata, Osaka, Japan
}

\section{Abstract}

Mouse monoclonal anti-Fas (CD95) antibody clone $\mathrm{CH}-11$ has been widely used in research on apoptosis. $\mathrm{CH}-11$ has the ability to bind to Fas protein on cell surface and induce apoptosis. Here, we used polystyrene beads coated with $\mathrm{CH}-11$ to investigate the role of lipid rafts in Fas-mediated apoptosis in SKW6.4 cells. Unexpectedly, by treatment of the cells with $\mathrm{CH}-11$-coated beads Fas protein was detached from cell surface and transferred to the surface of CH-11-coated beads. Western blot analysis showed that Fas protein containing both extracellular and intracellular domains was attached to the beads. Fas protein was not transferred from the cells to the surface of the beads coated with other anti-Fas antibodies or Fas ligand. Similar phenomenon was observed in Jurkat T cells. Furthermore, CH-11-induced apoptosis was suppressed by pretreatment with $\mathrm{CH}$-11-coated beads in Jurkat cells. These results suggest that $\mathrm{CH}-11$ might possess distinct properties on Fas protein compared with other anti-Fas antibodies or Fas ligand, and also suggest that caution should be needed to use polystyrene beads coated with antibodies such as CH-11.

\section{Introduction}

Fas (CD95) is a type I membrane protein and has a pivotal role in apoptosis. ${ }^{1}$ Antihuman Fas monoclonal antibody clone CH-11 was reported to induce apoptosis in $1989,{ }^{2}$ and thereafter Fas and Fas ligand (FasL) has been identified. ${ }^{3,4}$ Loss of function mutant mice of either Fas or FasL showed lymphoproliferative phenotype, demonstrating that FasL/Fas system has a critical role in the regulation of lymphocytes. ${ }^{5,6}$ Furthermore, it has been shown that FasL/Fas system is involved in the target cell lysis by cytotoxic $\mathrm{T}$ cells. ${ }^{\text {? }}$

The mechanisms by which FasL and Fas induce apoptosis have been extensively studied. ${ }^{1,8}$ Binding of FasL to Fas induces aggregation of Fas and Fas-associated proteins includ- ing FADD and caspase-8, which is termed death-inducing signaling complex (DISC) ${ }^{9}$ resulting in the activation of caspase cascade. Whether lipid rafts are involved in FasL/Fas system remains controversial. It was reported that DISC formed in lipid rafts and lipid rafts played an essential role in Fas-induced apoptosis. ${ }^{10}$ However, another report showed that lipid rafts were dispensable in Fas-induced apoptosis. ${ }^{11}$ In another report cholesterol depletion by methyl- $\beta$-cyclodextrin did not inhibit Fas association within lipid rafts, suggesting that Fas might be localized in sphingolipid-rich core of lipid rafts. ${ }^{12}$ In those studies agonistic anti-Fas antibodies were directly added to culture medium. To gain more insight on the role of lipid rafts in Fas-induced apoptosis, we tried to use polystyrene beads coated with anti-Fas antibody CH-11. Polystyrene beads coated with antibodies against surface proteins have been used to study lipid rafts..$^{13}$ As expected, incubation with $\mathrm{CH}-11$-coated beads induced apoptosis. However, it was unexpectedly observed that Fas protein was transferred from cell surface to the surface of $\mathrm{CH}$-11-coated beads after incubation of the cells with the beads.

\section{Materials and Methods}

\section{Cell culture}

SKW6.4 human B cell line or Jurkat human $\mathrm{T}$ cell line was cultured in RPMI 1640 containing $10 \%$ fetal bovine serum with $100 \mathrm{U} / \mathrm{mL}$ penicillin and $100 \mu \mathrm{g} / \mathrm{mL}$ streptomycin in a humidified $5 \% \mathrm{CO}_{2}$ incubator at $37^{\circ} \mathrm{C}$.

\section{$\mathrm{CH}-11$-coated beads}

Polybead polystyrene microsphere $6 \mu \mathrm{m}$ was purchased from Polysciences. The beads (20 $\mu \mathrm{L}$ ) were incubated with $1 \mu \mathrm{g}$ (or the indicated amounts) of mouse anti-Fas monoclonal antibody clone CH-11, clone ZB4 (MBL, Japan), clone 5E2, mouse IgM (Ancell Corporation), or SuperFasL (Alexis) in $100 \mu \mathrm{L}$ PBS overnight. Beads were washed twice with and suspended in $100 \mu \mathrm{L}$ PBS containing $1 \%$ albumin.

\section{Immunostaining and microscopy}

Cells $\left(5 \times 10^{5}\right)$ in $0.5 \mathrm{~mL}$ medium were incubated with $1 \mu \mathrm{L}$ FITC-conjugated anti-Fas antibody clone $5 \mathrm{E} 2$ for $30 \mathrm{~min}$, and further incubated with $10 \mu \mathrm{L}$ beads coated with CH-11, ZB4, $5 \mathrm{E} 2$, IgM or SuperFasL for the indicated times. Cells were washed with PBS and fixed in 4\% paraformaldehyde in PBS at room temperature for $10 \mathrm{~min}$. Cells were mounted on slides with Aqua Poly/Mount (Polysciences) and visualized with Fluoview FV300 confocal laser scanning microscope system (Olympus).
Correspondence: Hirofumi Sawai, Department of Internal Medicine, Osaka Dental University, 8-1 Kuzuhahanazonocho, Hirakata, Osaka 573-1121, Japan. Tel: +81.72.864.3079. Fax: +81.72.864.3179. E-mail: sawai@cc.osaka-dent.ac.jp

Key words: Fas; CD95; CH-11; apoptosis; Fas ligand; polystyrene beads.

Acknowledgments: this work was supported in part by "High-Tech Research Center" Project for Private Universities: matching fund subsidy from Ministry of Education, Culture, Sports, Science and Technology (2007-2011).

Received for publication: 2 November 2009. Accepted for publication: 7 January 2010.

This work is licensed under a Creative Commons Attribution 3.0 License (by-nc 3.0).

CC Copyright H. Sawai and N. Domae, 2010 Licensee PAGEPress, Italy

European Journal of Histochemistry 2010; $54: e 8$ doi:10.4081/ejh.2010.e8

\section{Separation of the cell fraction and} the beads fraction

Cells $\left(1 \times 10^{6}\right)$ in $0.5 \mathrm{~mL}$ medium were treated with $20 \mu \mathrm{L}$ CH-11-coated beads for $30 \mathrm{~min}$. Cells were washed with PBS, pelleted down and frozen at $-30^{\circ} \mathrm{C}$. Lysis buffer $(80 \mu \mathrm{L})$ containing $10 \mathrm{mM}$ Tris/HCl (pH7.4), $5 \mathrm{mM}$ EDTA and $1 \mathrm{mM}$ PMSF was added to the pellet. Cells were lysed by passing through 27 -gauge needle 20 times. The lysate was centrifuged at $800 \times \mathrm{g}$ for $5 \mathrm{~min}$ at $4^{\circ} \mathrm{C}$ to pellet down beads and cell nuclei. The supernatant containing cytosol and cell membrane was used as the cell fraction. Laemmli's sample buffer was added to the pellet containing beads, boiled for $2 \mathrm{~min}$, centrifuged at $10000 \times \mathrm{g}$ for $5 \mathrm{~min}$ at $4^{\circ} \mathrm{C}$ and used as the beads fraction.

\section{Western blot analysis}

The samples were separated in 10\% SDSPAGE and transferred onto Immobilon PVDF membrane (Millipore). The membrane was preincubated with PBS containing 0.1\% Tween 20 (PBS-T) plus 10\% Blocking-One (Nacalai Tesque, Japan) for $1 \mathrm{~h}$, and incubated with mouse monoclonal anti-Fas antibody clone 3D5 (Kamiya Biomedical Company), rabbit polyclonal anti-Fas antibody C-20, N-18, mouse monoclonal anti-Fas antibody B-10 (Santa Cruz Biotechnology), mouse monoclonal antiFADD antibody clone 1 (BD Biosciences), or mouse monoclonal anti-caspase- 8 antibody clone $1 \mathrm{C} 12$ (Cell Signaling Technology) for $2 \mathrm{~h}$. After washing with PBS-T for 15 and $5 \mathrm{~min}$, the membrane was further incubated with HRPconjugated anti-mouse or rabbit IgG antibody for $1 \mathrm{~h}$. The membrane was washed with PBS- 
$\mathrm{T}$ for 5 min three times and incubated with SuperSignal West Pico Chemiluminescent Substrate (Pierce) according to the manufacturer's instruction. The chemiluminescent image was visualized with VersaDoc 3000 imaging system (Bio-Rad).

\section{Caspase-3 activity assay}

Cells were lysed in lysis buffer containing 10 mM Hepes/KOH (pH 7.4), 1\% CHAPS, 5 mM EDTA and $1 \mathrm{mM}$ PMSF. After centrifugation at $10000 \times \mathrm{g}$ for $10 \mathrm{~min}$, the supernatant was used for the assay. The reaction buffer contained 10 mM Hepes/KOH (pH 7.4), 0.1\% CHAPS, 10\% sucrose, $1 \mathrm{mM}$ DTT and $10 \mu \mathrm{M}$ DEVD-MCA. Cell lysate $(10 \mu \mathrm{L})$ was mixed with $100 \mu \mathrm{L}$ reaction buffer in a 96 well plate. After $60 \mathrm{~min}$ incubation at $37^{\circ} \mathrm{C}$, fluorescence was detected using SpectraMax M5 (Molecular Devices) (excitation, $355 \mathrm{~nm}$; emission, $460 \mathrm{~nm}$ ).

\section{Results}

To investigate the role of lipid rafts in Fasmediated apoptosis, polystyrene beads were coated with anti-Fas antibody clone $\mathrm{CH}-11$ and human B cell line SKW6.4, in which Fas is highly expressed, was incubated with the beads. As expected, apoptosis was induced by overnight incubation of SKW6.4 cells with $\mathrm{CH}$ 11-coated beads but not by non-coated or control mouse IgM-coated beads (data not shown). Caspase- 3 activation was detected not within 1 $\mathrm{h}$ but after $2 \mathrm{~h}$ incubation of SKW6.4 cells with CH-11(0.1 $\mu$ g or more)-coated beads (data not shown). To visualize Fas aggregation, cells were stained with FITC-conjugated anti-Fas antibody (clone 5E2) and then treated with beads coated with $\mathrm{CH}-11$ (Figure $1 \mathrm{~A}$ ). In control experiments where the cells were treated with non-coated or IgM-coated beads, only the cell surface of SKW6.4 was fluorescently stained. Unexpectedly, fluorescence was observed not on the cell surface but on the surface of CH-11-coated beads after incubation of the cells with the beads. After 5 min incubation of the cells with the beads, slight fluorescence was observed on the surface of CH-11-coated beads with the residual fluorescence on cell surface. Fluorescence was observed almost exclusively on the beads after 30 min incubation. When the cells were first treated with $\mathrm{CH}$ 11-coated beads and then stained with FITClabeled anti-Fas antibody (5E2), the surface of CH-11-coated beads was similarly stained with fluorescence (data not shown). Similar staining of the surface of $\mathrm{CH}-11$-coated beads after 30 min incubation with the cells was observed using another anti-Fas antibody clone ZB4 instead of 5E2 (data not shown). The surface of CH-11-coated beads was not fluorescently
A

(-)
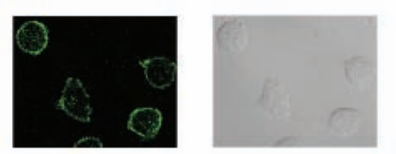

$\lg M$

$1 \mu \mathrm{g}$
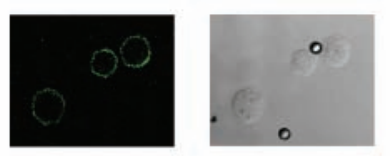

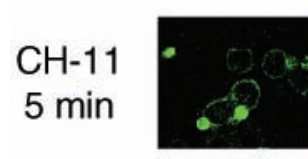

$\mathrm{CH}-11$
15 min

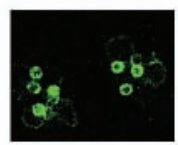

$\mathrm{CH}-11$ $30 \mathrm{~min}$

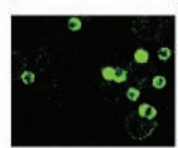

$\mathrm{CH}-11$

$60 \mathrm{~min}$

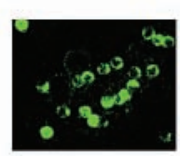

$\lg \mathrm{M}$ $60 \mathrm{~min}$

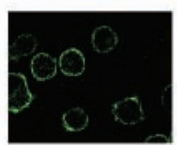

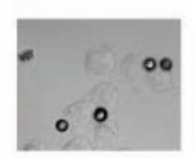

$\mathrm{CH}-11$ $0.25 \mu \mathrm{g}$
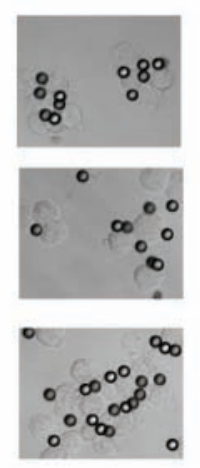
$\mathrm{CH}-11$ $1 \mu \mathrm{g}$
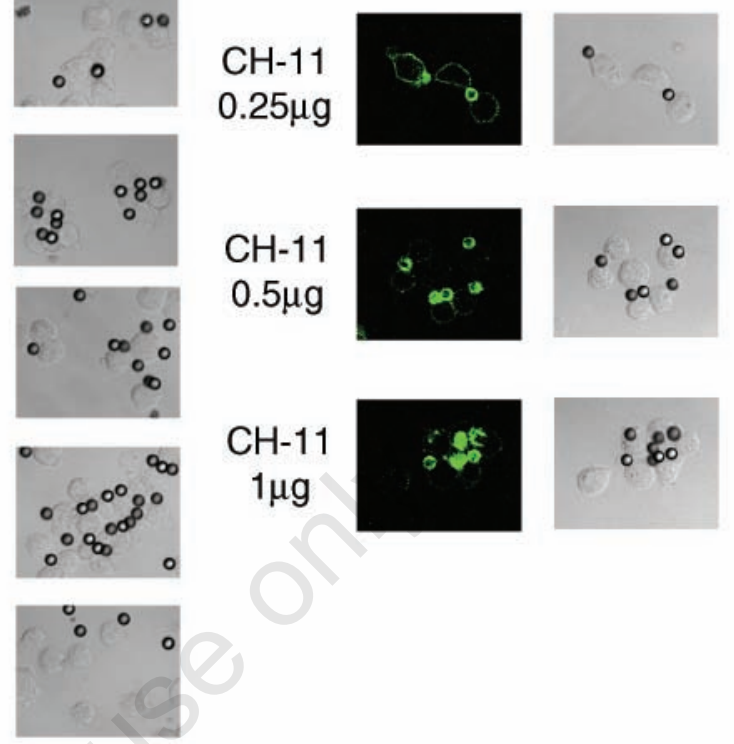

Figure 1. CH-11-coated beads were stained with FITC-conjugated anti-Fas antibody after incubation of SKW6.4 cells with the beads. (A) SKW6.4 cells were preincubated with FITC-conjugated anti-Fas antibody (5E2) for $30 \mathrm{~min}$ and then incubated with $\mathrm{CH}-11$ - or IgM-coated beads for the indicated times. (B) SKW6.4 cells were preincubated with FITC-conjugated anti-Fas antibody (5E2) for $30 \mathrm{~min}$ and then incubated for $60 \mathrm{~min}$ with the beads coated with the indicated amounts of $\mathrm{CH}-11$ or IgM as described in "Materials and Methods". Left, FITC; right, differential interference contrast (DIC).

stained when the beads were incubated without the cells, ruling out the possibility that $5 \mathrm{E} 2$ or ZB4 directly binds to the surface of $\mathrm{CH}-11$ coated beads. These results suggested that at least a part of Fas protein was transferred from the cell surface to the surface of $\mathrm{CH}$-11-coated beads after incubation of the cells with the beads. When the beads were coated with 0.25 $\mu \mathrm{g}$ of $\mathrm{CH}-11$ as described in "Materials and Methods", both the beads and the cell surface were fluorescently stained after 60 min incubation of the cells with the beads (Figure $1 \mathrm{~B}$ ). By increasing the amount of CH-11 (up to 1 $\mu \mathrm{g})$, fluorescence on the cell surface was reduced and that on the beads was increased, indicating dose-dependency of the phenomenon. Since it has been reported that extracellular domain of Fas can be cleaved by a matrix metalloproteinase (MMP),${ }^{14}$ the cells were pretreated with $10 \mu \mathrm{M}$ GM6001, a broad-spectrum MMP inhibitor, and then incubated with $\mathrm{CH}$ 11-coated beads. Again CH-11-coated beads were fluorescently stained (data not shown), suggesting that Fas protein was not cleaved by MMP under the experimental conditions.

To confirm that Fas was transferred to the
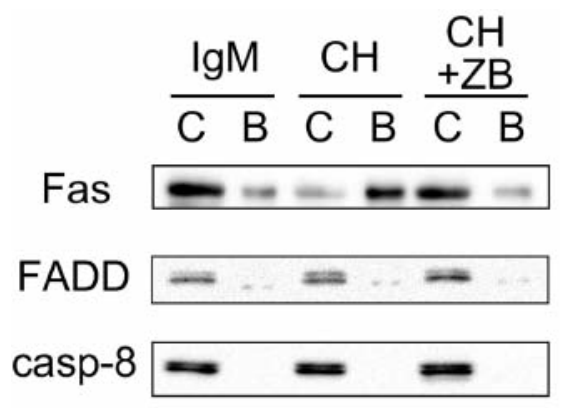

Figure 2. Fas protein was transferred from the cell surface to the surface of $\mathrm{CH}-11$ coated beads after incubation of the cells with the beads. SKW6.4 cells $\left(1 \times 10^{6}\right.$ in 0.5 mL medium) were treated with $20 \mu \mathrm{L}$ IgMcoated beads for $30 \mathrm{~min}$ (IgM), or treated with $20 \mu \mathrm{L} \mathrm{CH}-11$-coated beads for 30 min $(\mathrm{CH})$, or pretreated with $20 \mu \mathrm{L}$ ZB4 $(50 \mu \mathrm{g} / \mathrm{mL})$ for $15 \mathrm{~min}$ and treated with $\mathrm{CH}$-11-coated beads for $30 \mathrm{~min}(\mathrm{CH}+\mathrm{ZB})$. The cell fraction $(C)$ and the beads fraction (B) were separated and Western blot analysis was performed as described in Materials and Methods using anti-Fas antibody clone 3D5, anti-FADD antibody, or anti-caspase-8 antibody. 
surface of CH-11-coated beads after incubation of the cells with the beads, Western blot analysis was performed. After 30 min incubation of the cells with $\mathrm{CH}$-11-coated beads, the beads together with the cells were pelleted down and lysed in hypotonic buffer without detergent to minimize the dissociation of the protein attached to the beads. After centrifugation Laemmli's sample buffer was added to the pellet containing the beads and cell nuclei, boiled for $2 \mathrm{~min}$ for the dissociation of the protein from the beads and used as the beads fraction. The supernatant containing the cytosol and cell membrane was used as the cell fraction. Western blot analysis was performed using antibody against Fas death domain (clone 3D5). Most of Fas protein was detected in the cell fraction when treated with non-coated or mouse-IgM-coated beads, whereas Fas protein was almost exclusively detected in the beads fraction when the cells were treated with $\mathrm{CH}$ 11-coated beads (Figure 2). The faint band of Fas in the beads fraction of control was observed probably due to incomplete separation of the cell and the beads fractions. Similar results were obtained using other anti-Fas antibodies including C-20 and B-10 (both against C-terminus of Fas) instead of 3D5 (data not shown). Anti-Fas antibody N-18 (against $\mathrm{N}$-terminus) did not apparently detect Fas (data not shown), in accordance with the previous report describing that Fas could not be detected by antibodies against $\mathrm{N}$-terminus of Fas. ${ }^{15}$ Since $\mathrm{CH}-11$ recognizes extracellular domain of Fas whereas 3D5, C-20 or B-10 recognizes intracellular domain of Fas, these results demonstrated that Fas protein containing both extracellular and intracellular domains was transferred to the surface of $\mathrm{CH}$ 11-coated beads. It is known that DISC (deathinducing signaling complex) including Fas, FADD, and caspase- 8 is formed after Fas ligation. In our experiments, FADD and caspase- 8 remained in the cell fraction after treatment with $\mathrm{CH}$-11-coated beads (Figure 2), suggesting that Fas was transferred to the surface of CH-11-coated beads before DISC formation.

To confirm that transfer of Fas from the cell surface to the surface CH-11-coated beads depends on specific interaction between Fas and $\mathrm{CH}-11$, cells were pretreated with antagonistic anti-Fas antibody clone ZB4, which has been shown to inhibit $\mathrm{CH}-11$-induced apoptosis. ${ }^{16}$ Fluorescence on the surface of CH-11coated beads after incubation of the cells with the beads was suppressed by pretreatment with ZB4 in a dose-dependent manner (Figure 3). Furthermore, Western blot analysis showed that pretreatment with ZB4 attenuated transfer of Fas from the cell fraction to the beads fraction induced by CH-11-coated beads (Figure 2). These results indicated that ZB4 specifically inhibited transfer of Fas from the cell surface to $\lg M$
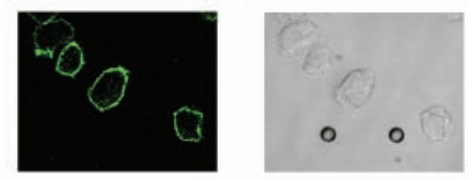

$\mathrm{CH}-11$
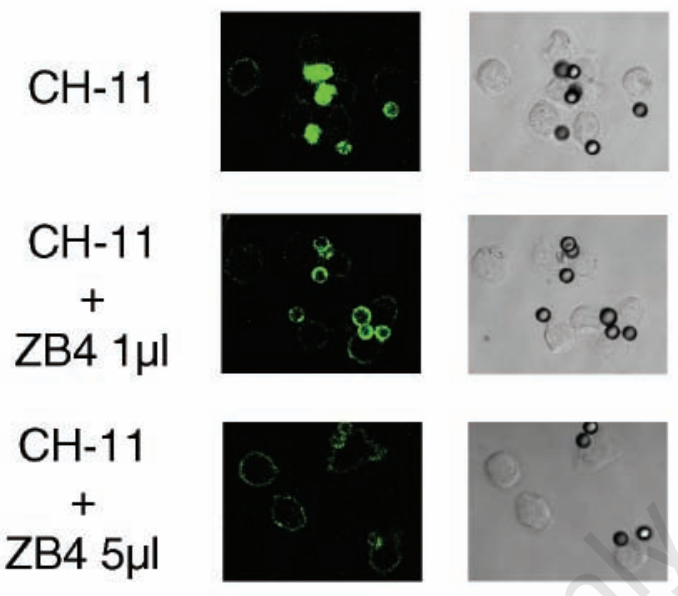

$(-)$
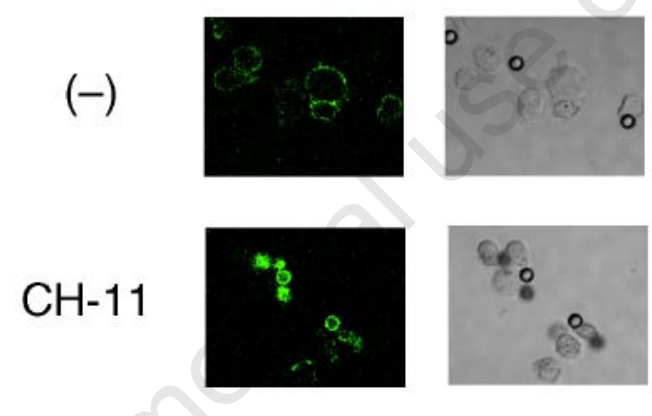

ZB4
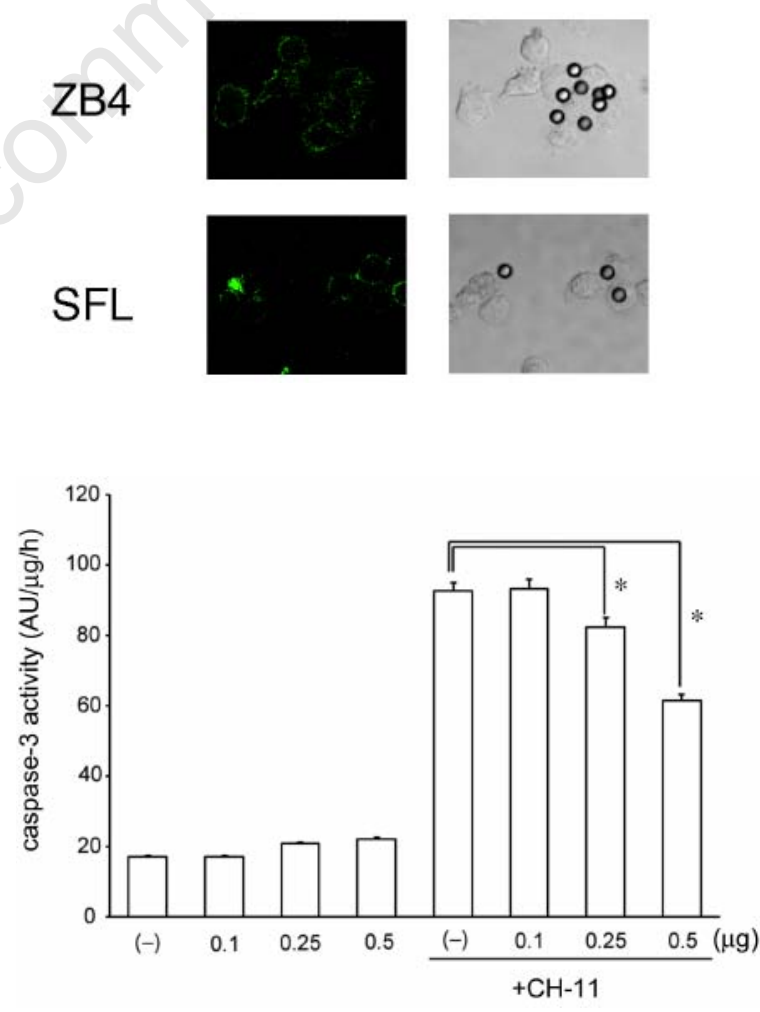

Figure 5. Preincubation of Jurkat cells with $\mathrm{CH}-11$ coated beads suppressed caspase- 3 activation induced by $\mathrm{CH}-11$ directly added to the cells. Jurkat cells were preincubated with the beads coated with the indicated amounts of $\mathrm{CH}-11$ as described in Materials and Methods for $30 \mathrm{~min}$, and then stimulated with 100 $\mathrm{ng} / \mathrm{mL} \mathrm{CH}-11$ for $90 \mathrm{~min}$. Caspase- 3 activity was measured as described in Materials and Methods. The data indicate means \pm SD $(\mathbf{n}=3)$. *, $\mathbf{P}<0.01$. 
the surface of CH-11-coated beads. Next, other anti-Fas antibodies (ZB4, 5E2) were used instead of $\mathrm{CH}-11$ to coat the beads. Overnight incubation of SKW6.4 cells with ZB4- or 5E2coated beads induced apoptosis, although less effectively than with $\mathrm{CH}-11$-coated beads (data not shown). When cells were incubated with FITC-conjugated 5E2 and then incubated with ZB4- or 5E2-coated beads, fluorescence was detected on the cell surface only and the surface of the beads was not fluorescently stained (Figure 4 and data not shown). To examine whether FasL could induce the similar phenomenon, beads were coated with SuperFasL (Alexis) and then the cells were incubated with FasL-coated beads. As expected, overnight incubation of SKW6.4 cells with FasL-coated beads induced apoptosis (data not shown). However, fluorescence was observed on the cell surface only and not on the surface of FasL-coated beads (Figure 4). These results further indicated that Fas was specifically transferred to $\mathrm{CH}$ 11-coated beads.

Moreover, similar phenomenon was observed in Jurkat T cells. When Jurkat cells were incubated with $\mathrm{CH}-11$-coated beads, Fas was transferred from the cell surface to the surface of CH-11-coated beads (data not shown). Furthermore, we investigated whether preincubation of Jurkat cells with CH-11-coated beads might inhibit apoptosis induced by $\mathrm{CH}-11$ directly added to the cells. As expected, preincubation of Jurkat cells with CH-11-coated beads suppressed caspase- 3 activation induced by $\mathrm{CH}$ 11 directly added to the cells (Figure 5), suggesting that transfer of Fas from the cell surface to the surface of $\mathrm{CH}-11$-coated beads inhibited apoptosis induced by CH-11 directly added to the cells. When similar experiments were performed using SKW6.4 cells, CH-11-induced caspase-3 activation was not significantly suppressed by pretreatment with $\mathrm{CH}-11$-coated beads. As described above, incubation with $\mathrm{CH}$ 11(0.1 $\mu \mathrm{g}$ or more)-coated beads induced caspase- 3 activation after $2 \mathrm{~h}$ in SKW6.4 cells. On the other hand, incubation of CH-11(0.5 $\mu \mathrm{g}$ or less)-coated beads for $2 \mathrm{~h}$ induced only slight caspase- 3 activation in Jurkat cells, presumably due to lower expression of Fas in Jurkat than in SKW6.4. Since preincubation of SKW6.4 with CH-11(0.25ug or less)-coated beads only partially transferred Fas from the cell surface to the beads (Figure $1 \mathrm{~B}$ ), $\mathrm{CH}-11$ directly added to the cells would induce ligation of the remaining Fas on the cell surface to activate caspase-3.

\section{Discussion}

CH-11 has been widely used as apoptosisinducing agonistic anti-Fas antibody. It was previously shown that Fas was internalized into the cells during apoptosis when agonistic anti-Fas antibody was directly added to culture medium. ${ }^{11}$ Here we unexpectedly found that Fas protein was transferred from the cell surface to the surface of CH-11-coated beads. Transfer of Fas was not observed by beads coated with FasL. In accordance with our results showing that agonistic anti-Fas antibody exerted different effects compared with FasL, another report showed that Fas microaggregates of approximately $250 \mathrm{kDa}$ formed in response to agonistic anti-Fas antibody but not to FasL. ${ }^{17}$ These results suggest that CH-11 (or other agonistic anti-Fas antibodies) may have distinct properties on Fas compared with FasL. Expression of soluble form of Fas lacking transmembrane domain has been reported. ${ }^{18}$ Although soluble form of Fas would bind to $\mathrm{CH}-11$-coated beads, the data presented in this paper indicated that Fas expressed on the cell surface was transferred to the surface of $\mathrm{CH}$-11-coated beads. Although Fas was almost completely transferred from the cell surface to the CH-11-coated beads within $1 \mathrm{~h}$ (Figure $1 \mathrm{~A}$ ), caspase-3 activation and apoptosis was induced by treatment with CH-11-coated beads in SKW6.4 cells, suggesting that apoptosis-inducing signaling was started immediately after Fas ligation by $\mathrm{CH}-11$-coated beads. Inhibition of $\mathrm{CH}$ 11-induced caspase-3 activation by preincubation with $\mathrm{CH}-11$-coated beads in Jurkat cells suggests that similar phenomenon might occur pathophysiologically. Thus, transfer of Fas from the cell surface by Fasbinding molecules would suppress Fas-mediated apoptosis. In conclusion, Fas protein was transferred from the cell surface to the surface of CH-11-coated beads not only in SKW6.4 but also in Jurkat cells. The precise mechanism remains unclear, but we suppose that the mechanism would be related to the specific interaction between Fas and CH-11. Fas-mediated apoptosis could be inhibited by preincubation with $\mathrm{CH}-11$-coated beads, suggesting that similar phenomenon might occur pathophysiologically. Also, caution should be needed to use polystyrene beads coated with antibodies such as CH-11.

\section{References}

1. Nagata S. Apoptosis by death factor. Cell 1997; 88:355-65.

2. Yonehara S, Ishii A, Yonehara M. A cellkilling monoclonal antibody (anti-Fas) to a cell surface antigen co-downregulated with the receptor of tumor necrosis factor. J Exp Med 1989; 169:1747-56.

3. Itoh N, Yonehara S, Ishii A, et al. The polypeptide encoded by the cDNA for human cell surface antigen Fas can mediate apoptosis. Cell 1991; 66:233-43.

4. Suda T, Takahashi T, Golstein P, Nagata S. Molecular cloning and expression of the Fas ligand, a novel member of the tumor necrosis factor family. Cell 1993; 75:116978.

5. Watanabe-Fukunaga R, Brannan CI, Copeland NG, et al. Lymphoproliferation disorder in mice explained by defects in Fas antigen that mediates apoptosis. Nature 1992; 356:314-7.

6. Takahashi T, Tanaka M, Brannan CI, et al. Generalized lymphoproliferative disease in mice, caused by a point mutation in the Fas ligand. Cell 1994; 76:969-76.

7. Nagata S, Golstein P. The Fas death factor. Science 1995; 267:1449-56.

8. Hengartner MO. The biochemistry of apoptosis. Nature 2000; 407:770-6.

9. Kischkel FC, Hellbardt S, Behrmann I, et al. Cytotoxicity-dependent APO-1 (Fas/CD95)-associated proteins form a death-inducing signaling complex (DISC) with the receptor. EMBO J 1995;14:557988.

10. Hueber A0, Bernard AM, Herincs Z, et al. An essential role for membrane rafts in the initiation of Fas/CD95-triggered cell death in mouse thymocytes. EMBO Rep 2002; 3:190-6.

11. Algeciras-Schimnich A, Shen L, Barnhart $\mathrm{BC}$, et al. Molecular ordering of the initial signaling events of CD95. Mol Cell Biol 2002; 22:207-20.

12. Eramo A, Sargiacomo M, Ricci-Vitiani L, et al. CD95 death-inducing signaling complex formation and internalization occur in lipid rafts of type I and type II cells. Eur J Immunol 2004; 34:1930-40.

13. Viola A, Schroeder S, Sakakibara Y, Lanzavecchia A. T lymphocyte costimulation mediated by reorganization of membrane microdomains. Science 1999; 283:680-2.

14. Strand S, Vollmer P, van den Abeelen L, et al. Cleavage of CD95 by matrix metalloproteinase-7 induces apoptosis resistance in tumour cells. Oncogene 2004; 23:3732-6.

15. Kamitani T, Nguyen HP, Yeh ET. 
Activation-induced aggregation and processing of the human Fas antigen. Detection with cytoplasmic domain-specific antibodies. J Biol Chem 1997; 272:22307-14.

16. Fadeel B, Thorpe CJ, Yonehara S, Chiodi F. Anti-Fas IgG1 antibodies recognizing the same epitope of Fas/AP0-1 mediate different biological effects in vitro. Int Immunol 1997; 9:201-9.

17. Legembre P, Beneteau M, Daburon S, et al. Cutting edge: SDS-stable Fas microaggregates: an early event of Fas activation occurring with agonistic anti-Fas anti- body but not with Fas ligand. J Immunol 2003;171:5659-62.

18. Cheng J, Zhou T, Liu C, Shapiro JP, Brauer MJ, Kiefer MC, et al. Protection from Fas-mediated apoptosis by a soluble form of the Fas molecule. Science 1994; 263:1759-62. 